Probability, Networks and Algorithms 
CWI is the National Research Institute for Mathematics and Computer Science. It is sponsored by the Netherlands Organization for Scientific Research (NWO).

CWI is a founding member of ERCIM, the European Research Consortium for Informatics and Mathematics.

CWI's research has a theme-oriented structure and is grouped into four clusters. Listed below are the names of the clusters and in parentheses their acronyms.

\section{Probability, Networks and Algorithms (PNA)}

Software Engineering (SEN)

Modelling, Analysis and Simulation (MAS)

Information Systems (INS)

Copyright (C) 2001, Stichting Centrum voor Wiskunde en Informatica

P.O. Box 94079, 1090 GB Amsterdam (NL)

Kruislaan 413, 1098 SJ Amsterdam (NL)

Telephone +31205929333

Telefax +31205924199

ISSN 1386-3711 


\title{
Exact Overflow Asymptotics for Queues with Many Gaussian Inputs
}

\author{
Krzysztof Dȩbicki \\ $C W I$ \\ P.O. Box 94079 \\ 1090 GB Amsterdam \\ The Netherlands \\ and \\ Mathematical Institute \\ University of Wroctaw \\ pl. Grunwaldzki 2/4 \\ 50-384 Wroctaw \\ Poland
}

\author{
Michel Mandjes \\ $C W I$ \\ P.O. Box 94079 \\ 1090 GB Amsterdam \\ The Netherlands \\ and \\ Faculty of Mathematical Sciences \\ University of Twente \\ P.O. Box 217 \\ 7500 AE Enschede \\ The Netherlands
}

\begin{abstract}
In this paper we consider a queue fed by a large number $n$ of independent continuoustime Gaussian processes with stationary increments. After scaling the buffer exceedance threshold $B$ and the (constant) service capacity $C$ by the number of sources (i.e., $B \equiv n b$ and $C \equiv n c$ ), we present asymptotically exact results for the probability that the buffer threshold is exceeded. We both consider the stationary overflow probability, and the (transient) probability of overflow at a finite time horizon $T$. We give detailed results on the practically important cases in which the inputs are fractional Brownian motion processes or integrated Gaussian processes.
\end{abstract}

2000 Mathematics Subject Classification: 60G15 (primary),60G70,60K25 (secondary). Keywords and Phrases: Gaussian processes, fluid queues, extremes, overflow probabilities, exact asymptotics, integrated Gaussian processes, fractional Brownian motion.

Note: Work carried out under the CWI project P1201. K. Dẹbicki was also supported by KBN under grant 5 P03A 02120 (2001-2003). 


\section{Introduction}

Recent studies indicate that Gaussian processes are a natural choice to model traffic in communication networks [1]. In for instance [7, 14] it was proved that, under heavy traffic environment parameterization, large number of i.i.d. on-off sources may be approximated by a Gaussian process (with the same covariance structure as the on-off process). Moreover Gaussian processes are endowed with the versatility to incorporate a broad range of correlation structures and still allow, to some level, explicit analysis. In this sense they are an attractive compromise. Importantly, fractional Brownian motion (FBM) received a lot of attention, since it has been proposed to model long-range dependent traffic with a self-similar structure, see e.g. [21].

An element in a communication network is usually modeled as a queue, fed by some input process, and emptied at a constant service rate $C$. Exact analyses of the buffer content distribution for Gaussian input processes are restricted to a few special cases, such as for queues of which the input is Brownian motion or Brownian bridge, see e.g. [24]. For other relevant source models, such as fractional Brownian motion, the queue length distribution is not available. This motivates the interest in simulation methods and asymptotical techniques; this paper focuses on the latter.

Asymptotics for tail probabilities. So far most of the asymptotic studies have focused on the large-buffer regime. More precisely, one is interested in approximations for large $B$ of $\mathbb{P}\left(Q^{\star}>B\right)$, being the probability that the steady-state queue length exceeds level $B$. Duffield \& O'Connell [11] proved for the special case of FBM input that $\log \left(\mathbb{P}\left(Q^{\star}>B\right)\right)$ is asymptotically (for large $B$ ) proportional to $B^{2(1-H)}$, with a proportionality constant $I$ that depends on service rate $C$ and Hurst parameter $H$. This type of asymptotics is known as logarithmic asymptotics. It says that $\mathbb{P}\left(Q^{\star}>B\right)=f(B) \exp \left(-I B^{2(1-H)}\right)$, with an (unknown) function $f(\cdot)$ with the property $\log f(B)=o\left(B^{2(1-H)}\right)$ as $B \rightarrow \infty$. Obviously, logarithmic asymptotics offer important qualitative insight, but the fact that $f(\cdot)$ is unknown makes it less useful for practical purposes. This motivates the research on so-called exact asymptotics; these provide us with a function $g(\cdot)$ such that $\mathbb{P}\left(Q^{\star}>B\right) / g(B) \rightarrow 1$, for $B \rightarrow \infty$ (which we will denote by $\mathbb{P}\left(Q^{\star}>B\right) \sim g(B)$ ). Exact asymptotics for queues with FBM input were derived by Hüsler \& Piterbarg [12], and Narayan [20]. For Gaussian input process with short range dependence structure exact asymptotics for $\mathbb{P}\left(Q^{\star}>B\right)$ as $B \rightarrow \infty$ were found in [6]; see also [9] and the references therein.

Many-sources asymptotics. An important limitation of large-buffer regime is that it does not provide us with insight into overflow behavior for small or moderately-sized buffers; this regime might be relevant in many practical situations. Another crucial observation is that often the input traffic stream can be seen as the superposition of many i.i.d. streams. These thoughts led to the idea of investigating the so-called many-sources regime. In this regime $n$ sources feed into the queue; the queueing resources buffer and bandwidth are scaled accordingly: $B \equiv n b$ and $C \equiv n c$, respectively. Under this scaling and fairly general conditions on the input process, for general $b$, the steady-state overflow probability decays exponentially in $n$. Logarithmic asymptotics were derived by Botvich \& Duffield 
[4], see also Courcoubetis \& Weber [5] and Simonian \& Guibert [25], for both discretetime and continuous-time queues. Exact asymptotics in discrete-time queues were found by Likhanov \& Mazumdar [16].

Contribution. In discrete time, the derivation of asymptotically exact expansions for the many-sources regime relies on the fact that there is a unique epoch, say $t^{\star} \in \mathbb{N}$, at which (with overwhelming probability) the overflow occurs, see e.g. [16]. Put differently, the asymptotics of the stationary overflow probability coincide with the probability of overflow at $t^{\star}$ (given that the system started empty at time 0 ). Importantly, this reasoning does not apply to continuous time: there the probability mass around the most likely epoch does contribute to the asymptotics. This makes the derivation of exact asymptotics in continuous-time more involved.

In this paper we find exact asymptotics of the overflow probability in queues with many Gaussian sources. The techniques used rely on earlier results by Piterbarg \& Prisyazhnyuk [23]. In addition to the asymptotics of the stationary workload, we also deal with transient probabilities, i.e., the probability that the buffer level exceeds $n b$ at some specific 'time horizon' $T$. It is noted that if $T>t^{\star}$, then the asymptotics of the stationary and transient probabilities are identical. Also, as can be expected, if $T<t^{\star}$, then most likely epoch of overflow is $T$ itself. We will rigorize these ideas in this paper.

Organization. The organization of this paper is as follows. In Section 2 we set up the framework for our analysis: we give a model description, we list the necessary assumptions, and we discuss relevant preliminaries. Section 3 is an overview of the results of this paper: the exact asymptotics for the transient and steady-state overflow probabilities. Section 4 presents the asymptotics for the practically important special cases of FBM input and Integrated Gaussian input. Section 5 gives the proofs of our results.

\section{Model description and preliminaries}

In this section we set up the framework for our analysis: we specify the traffic model and list the assumptions. We also motivate the relevance of our study, and relate our results to previous work.

\section{$2.1 \quad$ Traffic model}

Let $\eta(\cdot) \equiv\left(\eta_{i}(t)\right)_{t \in \mathbb{R}}$ be a centered Gaussian stochastic process with stationary increments, continuous sample paths a.s., with $\eta(0)=0$ and the standard deviation function

$$
\sigma_{\eta}(t):=\sqrt{\operatorname{Var}(\eta(t))} .
$$

By $\eta_{i}(\cdot), i=1, \ldots, n$, we denote a sequence of i.i.d. copies of $\eta(\cdot)$.

In this paper we consider a fluid queue fed by the superposition of $n$ i.i.d. sources $\eta_{1}(\cdot), \ldots, \eta_{n}(\cdot)$, where $\eta_{i}(\cdot)$ describes the cumulative amount of traffic generated by $i$-th source, $i=1, \ldots, n$, in time interval $[0, t]$. We assume that the queue has a constant drain rate $C$. 
In classical papers on fluid queues, traffic is usually modeled as a stationary alternating on-off process; see e.g. [2], where the on- and off-times are assumed to be exponentially distributed. The analysis of queues with Gaussian input is motivated by a central limit theorem type of argumentation. Under heavy traffic environment parameterization, large number of on-off sources may be well approximated by a Gaussian process with the same covariance function as the covariance function of the generic on-off source, see [7, 14]; cf. also [1]. A technical drawback is that the Gaussianity of the input processes in principle allows 'negative traffic'; this is however of minor impact, see for instance [1].

Given the applications in communication networks, the following special cases of $\eta(\cdot)$ play an important role:

- Fractional Brownian motion (FBM) case: $\eta(t)=B_{H}(t)$, where $B_{H}(\cdot)$ is the fractional Brownian motion with Hurst parameter $H \in(0,1)$; that is, $\eta(\cdot)$ is a centered Gaussian process with stationary increments, continuous sample paths, $\eta(0)=0$ a.s., and variance function $\sigma_{\eta}^{2}(t)=t^{2 H}$.

- Integrated Gaussian (IG) case: $\eta(t)=\int_{0}^{t} Z(s) \mathrm{d} s$, where $Z(\cdot)$ is a centered stationary Gaussian process with continuous covariance function $R(t)=\operatorname{Cov}(Z(s+t), Z(s))$.

The FBM case is motivated by statistical analyses of network traces, where self-similarity and long range dependence property were observed, e.g. [15]. The relevance of the IG case is motivated in $[8,14,26]$, see also survey [9].

\subsection{Assumptions}

In this paper we make the following assumptions on the standard deviation function of the generic process $(\eta(t))_{t \in \mathbb{R}}$.

A1 The function $\sigma_{\eta}(\cdot)$ is 'sublinear': $\lim _{t \rightarrow \infty} \sigma_{\eta}(t) / t=0$;

A2 The function $\sigma_{\eta}(\cdot) \in C^{2}([0, \infty))$ is strictly increasing and strictly concave;

A3 The function behaves as a polynomial for small $t$ : for some constant $A_{0}>0$ and $\gamma>0$

$$
\lim _{t \downarrow 0} \frac{\sigma_{\eta}^{2}(t)}{t^{\gamma}}=A_{0} .
$$

To short the notation, if $(\eta(t))_{t \in \mathbb{R}}$ satisfies $\mathbf{A 1 - A 3 , ~ t h e n ~ w e ~ w i l l ~ w r i t e ~} \eta(\cdot) \in \mathbf{A} 1-\mathbf{A 3}$.

\subsection{Preliminaries}

Assuming that there the is $Q(0)=0$ fluid in the queue at time $t=0$, the transient probability distribution that at time $T>0$ the amount of fluid in the queue $Q(T)$ exeeds threshold $B$ has the following representation:

$$
\pi_{n}(T) \equiv \mathbb{P}(Q(T)>B)=\mathbb{P}\left(\sup _{t \in[0, T]} \sum_{i=1}^{n} \eta_{i}(t)-C t>B\right) .
$$

Analogously, the stationary overflow probability equals

$$
\pi_{n} \equiv \mathbb{P}\left(Q^{\star}>B\right)=\mathbb{P}\left(\sup _{t \in[0, \infty)} \sum_{i=1}^{n} \eta_{i}(t)-C t>B\right) .
$$


Unfortunately, so far for general Gaussian processes no explicit expressions for (2.1) and (2.2) have been found - results are available only for specific cases, such as Brownian motion and Brownian bridge, see e.g. [24, Eq. (2.2.8)]. Therefore, research has focused on asymptotics, in particular large buffer asymptotics, that is for $B \rightarrow \infty$; we refer to $[12,19,20,21]$ for FBM input, and $[6,8,10,14,26]$ for IG traffic.

In this paper we examine another asymptotic regime, namely the regime in which the number of sources grows large. We rescale the buffer threshold and link capacity with the number of sources: $B \equiv n b$ and $C \equiv n c$. This regime, introduced in the seminal paper by Weiss [27], leads itself to asymptotic analysis. It turned out that under very general conditions the stationary overflow probability $\pi_{n}$ decays exponentially in $n$, where the decay rate $I(b, c)$ is given by

$$
\begin{aligned}
-\lim _{n \rightarrow \infty} \frac{1}{n} \log \pi_{n}:=I(b, c) & =\inf _{t \in \mathbb{R}_{+}} \sup (\theta(b+c t)-\log \mathbb{E} \exp (\theta \eta(t))) \\
& =\inf _{t \in \mathbb{R}_{+}} \sup _{\theta}\left(\theta(b+c t)-\frac{1}{2} \theta^{2} \sigma_{\eta}^{2}(t)\right) \\
& =\inf _{t \in \mathbb{R}_{+}} \frac{m^{2}(t)}{2}, \text { with } m(t):=\frac{b+c t}{\sigma_{\eta}(t)}
\end{aligned}
$$

see Botvich \& Duffield [4]. The drawback of this result is that it provides us only with logarithmic asymptotics and does not give us a function $g(\cdot)$ such that $\pi_{n} / g(n) \rightarrow 1$ as $n \rightarrow \infty$. For the relevant case of on-off sources, the decay rate $I(b, c)$ can be given quite explicitly, see $[17,18]$.

In discrete time, exact asymptotics were found, see e.g. Likhanov \& Mazumdar [16]. The above continuous-time relation for the decay rate still holds, but with the infimum over $\mathbb{N}$ rather than $\mathbb{R}_{+}$. Calling the optimizing argument in (2.3) $t^{\star}$, we get that

$$
\pi_{n} \equiv \mathbb{P}\left(\sup _{t \in \mathbb{N}} \sum_{i=1}^{n} \eta_{i}(t)-C t>B\right) \sim \Psi\left(m\left(t^{\star}\right) \sqrt{n}\right)
$$

as $n \rightarrow \infty$ with

$$
\Psi(x):=\int_{x}^{\infty} \frac{1}{\sqrt{2 \pi}} e^{-\frac{1}{2} y^{2}} \mathrm{~d} y
$$

being the complementary standard normal distribution function. Let us recall that $\Psi(\cdot)$ obeys the following asymptotic equivalence:

$$
\Psi(x) \sim \frac{1}{x \sqrt{2 \pi}} e^{-\frac{1}{2} x^{2}} .
$$

The exact asymptoics given in (2.4) can be interpreted as follows. With the Bahadur-Rao [3] result, it follows that the asymptotically exact expression in (2.4) is the same for

$$
\mathbb{P}\left(\sum_{i=1}^{n} \eta_{i}\left(t^{\star}\right)-n c t^{\star}>n b\right) .
$$

So $t^{\star}$ can be interpreted as the most likely epoch of overflow; apparently only the probability mass in $t^{\star}$ plays (for large $n$ ) as significant role, in that we can neglect the probability of overflow at other epochs. 
In continuous time, there is again the notion of most likely epoch of overflow. However, now the probability mass around this epoch cannot be neglected, if we are interested in asymptotically exact rather than logarithmic asymptotics. This makes the analysis of the continuous-time case more involved. In this paper we rely on techniques developed by Piterbarg \& Prisyazhnyuk [23] to find asymptotically exact asymptotics.

\section{$3 \quad$ Exact asymptotics}

In this section we present the results of this paper. Proofs are provided in Section 5. Our results consist of the exact asymptotics of (2.1) and (2.2) as $n \rightarrow \infty$. The asymptotics of the transient probability $\pi_{n}(T)$ strongly depends on the play between value of the time horizon $T$ and the optimizing argument $t^{\star}$ in (2.3). We therefore distinguish between three cases: (1) $T>t^{\star}$, (2) $T=t^{\star}$, (3) $T<t^{\star}$. The stationary probability $\pi_{n}$ coincides with the case $T>t^{\star}$ (as it can be interpreted as $T \equiv \infty$ ).

Remark 3.1 As follows directly from (2.3), $t^{\star}$ is determined by

$$
c \sigma_{\eta}\left(t^{\star}\right)=\left(b+c t^{\star}\right) \sigma_{\eta}{ }^{\prime}\left(t^{\star}\right) .
$$

In the following lemma we show that under A1-A3 equation (3.6) has a unique root.

Lemma 3.1 Let $\eta(\cdot) \in \mathbf{A} 1-\mathbf{A 3}$. Then $m^{\prime}(t)=0$ has exactly one root, say $t^{\star}$. Moreover, $m(t)$ is strictly decreasing on $t \in\left(0, t^{\star}\right)$ and strictly increasing on $t \in\left(t^{\star}, \infty\right)$.

Proof. Define $\phi(t):=\sigma_{\eta}(t) / \sigma_{\eta}{ }^{\prime}(t)-t$. Since

$$
m^{\prime}(t)=\frac{c \sigma_{\eta}(t)-(c t+b) \sigma_{\eta}{ }^{\prime}(t)}{\sigma_{\eta}^{2}(t)},
$$

it suffices to prove that for each $b, c>0$

$$
\phi(t)=\frac{b}{c}
$$

has a unique root $t^{\star}$ and $\phi(\cdot)$ is strictly increasing.

Due to A1 it follows that $\lim _{t \rightarrow 0} m(t)=\infty$ and $\lim _{t \rightarrow \infty} m(t)=\infty$ for each $b, c>0$. Thus equation (3.7) has at least one solution. Moreover

$$
\phi^{\prime}(t)=\frac{\left(\sigma_{\eta}{ }^{\prime}(t)\right)^{2}-\sigma_{\eta}(t) \sigma_{\eta}{ }^{\prime \prime}(t)}{\left(\sigma_{\eta}{ }^{\prime}(t)\right)^{2}}-1=-\frac{\sigma_{\eta}(t) \sigma_{\eta}{ }^{\prime \prime}(t)}{\left(\sigma_{\eta}{ }^{\prime}(t)\right)^{2}}>0,
$$

since $\sigma_{\eta}^{\prime \prime}(t)<0$ due to the strict concavity, cf. A2. Thus $\phi(\cdot)$ is strictly increasing. This completes the proof.

In the asymptotics presented below so-called Pickands constants [22] play a crucial role; they are defined by the following limit:

$$
\mathcal{H}_{\alpha}:=\lim _{T \rightarrow \infty} \frac{1}{T} \cdot \mathbb{E} \exp \left(\sup _{t \in[0, T]}\left(\sqrt{2} B_{\alpha / 2}(t)-t^{\alpha}\right)\right),
$$


where $\alpha \in(0,2]$.

In case (1) the most likely epoch of overflow (in the infinite-horizon system) $t^{\star}$ lies before time horizon $T$. Heuristically, this says that, on $[0, T]$, overflow will occur most likely at time $t^{\star}$. We get the following result.

Theorem 3.2 Let $\eta(\cdot) \in \mathbf{A} 1-\mathbf{A} 3$ and $T>t^{\star}$.

(i) If $0<\gamma<2$, then, for $n \rightarrow \infty$,

$$
\pi_{n}(T) \sim \frac{\mathcal{H}_{\gamma}}{\sqrt{\pi}}\left(\frac{2 \sigma_{\eta}\left(t^{\star}\right)}{-\sigma_{\eta}{ }^{\prime \prime}\left(t^{\star}\right)}\right)^{1 / 2}\left(\frac{A_{0}}{2 \sigma_{\eta}^{2}\left(t^{\star}\right)}\right)^{1 / \gamma}\left(m\left(t^{\star}\right)\right)^{\frac{2}{\gamma}-1} \cdot n^{\frac{1}{\gamma}-\frac{1}{2}} \Psi\left(m\left(t^{\star}\right) \sqrt{n}\right) .
$$

(ii) If $\gamma=2$, then, for $n \rightarrow \infty$,

$$
\pi_{n}(T) \sim \sqrt{1+\frac{\left(\sigma_{\eta}{ }^{\prime}\left(t^{\star}\right)\right)^{2}-\left(\sigma_{\eta}{ }^{\prime}(0)\right)^{2}}{\sigma_{\eta}\left(t^{\star}\right) \sigma_{\eta}{ }^{\prime \prime}\left(t^{\star}\right)}} \cdot \Psi\left(m\left(t^{\star}\right) \sqrt{n}\right) .
$$

In case (2) the most likely epoch of overflow (in the-infinite-horizon system) $t^{\star}$ and the time horizon $T$ coincide. Therefore, on $[0, T]$ overflow will occur most likely at time $T$. It can be expected that, given overflow in the infinite-horizon system, half of the probability mass will be before $T$. This heuristically explains the following result (cf. Theorem 3.2).

Theorem 3.3 Let $\eta(\cdot) \in \mathbf{A} 1-\mathbf{A} 3$ and $T=t^{\star}$.

(i) If $0<\gamma<2$, then, for $n \rightarrow \infty$,

$$
\pi_{n}(T) \sim \frac{\mathcal{H}_{\gamma}}{2 \sqrt{\pi}}\left(\frac{2 \sigma_{\eta}(T)}{-\sigma_{\eta}{ }^{\prime \prime}(T)}\right)^{1 / 2}\left(\frac{A_{0}}{2 \sigma_{\eta}^{2}(T)}\right)^{1 / \gamma}(m(T))^{\frac{2}{\gamma}-1} \cdot n^{\frac{1}{\gamma}-\frac{1}{2}} \Psi(m(T) \sqrt{n}) .
$$

(ii) If $\gamma=2$, then, for $n \rightarrow \infty$,

$$
\pi_{n}(T) \sim \frac{1}{2} \sqrt{1+\frac{\left(\sigma_{\eta}^{\prime}(T)\right)^{2}-\left(\sigma_{\eta}^{\prime}(0)\right)^{2}}{\sigma_{\eta}(T) \sigma_{\eta}{ }^{\prime \prime}(T)}} \cdot \Psi(m(T) \sqrt{n}) .
$$

In case (3) the most likely time of exceeding buffer level $n b$ (in the infinite-horizon system) $t^{\star}$ majorizes the horizon $T$. Therefore, the most likely time of overflow in time interval $[0, T]$ is $T$. The following result describes the asymptotics for this case.

Theorem 3.4 Let $\eta(\cdot) \in \mathbf{A} 1-\mathbf{A 3}$ and $T<t^{\star}$.

(i) If $0<\gamma<1$, then, for $n \rightarrow \infty$,

$$
\pi_{n}(T) \sim \mathcal{H}_{\gamma}\left(\frac{A_{0}}{2 \sigma_{\eta}^{2}(T)}\right)^{1 / \gamma}\left(\frac{(m(T))^{\frac{2}{\gamma}-1}}{-m^{\prime}(T)}\right) \cdot n^{\frac{1}{\gamma}-1} \Psi(m(T) \sqrt{n}) .
$$

(ii) If $\gamma=1$, then, for $n \rightarrow \infty$,

$$
\pi_{n}(T) \sim\left(1-\frac{A_{0}}{2 \sigma_{\eta}^{2}(T)} \frac{m(T)}{m^{\prime}(T)}\right) \cdot \Psi(m(T) \sqrt{n}) .
$$

(iii) If $1<\gamma \leq 2$, then, for $n \rightarrow \infty$,

$$
\pi_{n}(T) \sim \Psi(m(T) \sqrt{n}) .
$$


As motivated above, the asymptotics of the stationary overflow probability $\pi_{n}$ coincides with case (1): $T>t^{\star}$.

Theorem 3.5 Let $\eta(\cdot) \in \mathbf{A} 1-\mathbf{A 3}$, and $T>t^{\star}$. Then, for $n \rightarrow \infty$,

$$
\pi_{n}(T) \sim \pi_{n}
$$

Due to (2.5), the above results implies that, for $T \geq t^{\star}$, the transient overflow probability $\pi_{n}(T)$ is asymptotically proportional to $n^{1 / \gamma-1} \exp \left(-n m^{2}\left(t^{\star}\right) / 2\right.$ ) (as a function of $n$ ). This type of asymptotics also holds in the stationary regime, i.e., for $\pi_{n}$. Notice that this is essentially different from the discrete-time asymptotics (2.4), which are of the form $n^{-1 / 2} \exp \left(-n m^{2}\left(t^{\star}\right) / 2\right)$. Noticing that $\gamma \in(0,2]$, this qualitative argument confirms the evident property that the overflow probabilities in continuous-time are larger than in discrete time. In fact, our continuous-time result gives insight into the probability of excursions between the gridpoints exceeding $n b$.

For $T<t^{\star}$, the transient overflow probability $\pi_{n}(T)$ resembles $n^{1 / \gamma-1 / 2} \exp \left(-n m^{2}\left(t^{\star}\right) / 2\right)$ for $0<\gamma \leq 1$, and $n^{-1 / 2} \exp \left(-n m^{2}\left(t^{\star}\right) / 2\right)$ for $1<\gamma \leq 2$.

\section{Special cases}

In this section we consider two specific, practically relevant, input processes: fractional Brownian motion (FBM) sources and integrated Gaussian (IG) sources.

\subsection{Fractional Brownian motion sources}

In the following propositions we give the exact asymptotic both for $\pi_{n}(T)$ and $\pi_{n}$ as $n \rightarrow \infty$ for fluid queues fed by superposition of many FBM sources. It is easily seen that assumptions A1-A3 are met, with $\gamma \equiv 2 H$ and $A_{0} \equiv 1$. It is straightforward to derive

$$
t^{\star}=\frac{H}{1-H} \cdot \frac{b}{c}
$$

and

$$
m:=m\left(t^{\star}\right)=\left(\frac{b}{1-H}\right)^{1-H}\left(\frac{c}{H}\right)^{H} .
$$

It is now a matter of inserting these expressions in the formulas of Theorems 3.2-3.4. This leads to the following results.

Proposition 4.1 Let $\eta(\cdot)$ be FBM input.

(i) If $T>t^{\star}$, then

$$
\pi_{n}(T) \sim \frac{\mathcal{H}_{2 H}}{\pi} \cdot \frac{1}{\sqrt{H(1-H)}} \cdot\left(\frac{m}{\sqrt{2}}\right)^{\frac{1}{H}-1} \cdot n^{1 / 2 H-1 / 2} \Psi(m \sqrt{n}), \quad \text { as } \quad n \rightarrow \infty ;
$$

(ii) If $T=t^{\star}$, then

$$
\pi_{n}(T) \sim \frac{\mathcal{H}_{2 H}}{2 \pi} \cdot \frac{1}{\sqrt{H(1-H)}} \cdot\left(\frac{m}{\sqrt{2}}\right)^{\frac{1}{H}-1} \cdot n^{1 / 2 H-1 / 2} \Psi(m \sqrt{n}), \quad \text { as } \quad n \rightarrow \infty ;
$$


(iii) If $T<t^{\star}$ and $H \in(0,1 / 2)$, then

$$
\pi_{n}(T) \sim \mathcal{H}_{2 H} \cdot \frac{T^{2 H-1}(b+c T)^{1 / H-1}}{c T-H(b+c T)} \cdot \frac{n^{1 / 2 H-1}}{2^{1 / 2 H}} \Psi\left(\frac{b+c T}{T^{H}} \cdot \sqrt{n}\right), \quad \text { as } n \rightarrow \infty ;
$$

(iv) If $T<t^{\star}$ and $H=1 / 2$, then

$$
\pi_{n}(T) \sim\left(1-\frac{1}{2 T^{2 H-1}} \cdot \frac{b+c T}{c T-H(b+c T)}\right) \cdot \Psi\left(\frac{b+c T}{T^{H}} \cdot \sqrt{n}\right), \quad \text { as } n \rightarrow \infty ;
$$

(v) If $T<t^{\star}$ and $H \in(1 / 2,1)$, then

$$
\pi_{n}(T) \sim \Psi\left(\frac{b+c T}{T^{H}} \cdot \sqrt{n}\right), \quad \text { as } n \rightarrow \infty .
$$

Combination of Theorem 3.5 with Proposition 4.1 gives the following asymptotics for the stationary buffer overflow.

Proposition 4.2 Let $\eta(\cdot)$ be FBM input. Then

$$
\pi_{n} \sim \frac{\mathcal{H}_{2 H}}{\pi} \cdot \frac{1}{\sqrt{H(1-H)}} \cdot\left(\frac{m}{\sqrt{2}}\right)^{\frac{1}{H}-1} \cdot n^{1 / 2 H-1 / 2} \Psi(m \sqrt{n}) \text {, as } n \rightarrow \infty .
$$

\subsection{Integrated Gaussian sources}

In this section we focus on the IG input process. Recall that in this case $\eta(\cdot)$ has the following form $\eta(t)=\int_{0}^{t} Z(s) \mathrm{d} s$, where $Z(\cdot)$ is a centered stationary Gaussian stochastic process with covariance function $R(\cdot)$. We make the following assumptions on $R(\cdot)$ :

IG1 $R(\cdot) \in C^{1}([0, \infty))$ and $R(t)>0$ for all positive $t$;

IG2 $R(\cdot)$ is strictly decreasing;

IG3 $\lim _{t \rightarrow \infty} R(t)=0$.

Since $\eta(\cdot)$ is IG, it follows that

$$
\sigma_{\eta}^{2}(t)=2 \int_{0}^{t} \mathrm{~d} s \int_{0}^{s} R(v) \mathrm{d} v .
$$

In view of (3.6) the most likely epoch of overflow $t^{\star}$ may be found as the unique solution of

$$
2 c \int_{0}^{t} \mathrm{~d} s \int_{0}^{s} R(v) \mathrm{d} v=(c t+b) \int_{0}^{t} R(v) \mathrm{d} v .
$$

In the following proposition we present the exact asymptotics of transient overflow probabilities for IG input processes $\eta(\cdot)$ satisfying IG1-IG3. The results are in terms of the covariance function $R(\cdot)$.

We use the notation $\dot{\sigma}_{\eta}^{2}(t)$ and $\ddot{\sigma}_{\eta}^{2}(t)$ for the first and second derivative of $\sigma_{\eta}^{2}(t)$ respectively. Notice that we have

$$
\sigma_{\eta}^{\prime}(t)=\frac{\dot{\sigma}_{\eta}^{2}(t)}{2 \sqrt{\sigma_{\eta}^{2}(t)}} \text { and } \sigma_{\eta}^{\prime \prime}(t)=\frac{1}{4} \frac{2 \ddot{\sigma}_{\eta}^{2}(t) \sigma_{\eta}^{2}(t)-\left(\dot{\sigma}_{\eta}^{2}(t)\right)^{2}}{\left(\sigma_{\eta}^{2}(t)\right)^{3 / 2}}
$$


for each $t \geq 0$. Also

$$
\dot{\sigma}_{\eta}^{2}(t)=2 \int_{0}^{t} R(v) \mathrm{d} v, \quad \ddot{\sigma}_{\eta}^{2}(t)=2 R(t), \quad \dddot{\sigma}_{\eta}^{2}(t)=2 R^{\prime}(t),
$$

where $R^{\prime}(\cdot)$ exists due to IG1.

Proposition 4.3 Let $\eta(\cdot)$ be IG input such that IG1-IG3 are satisfied.

(i) If $T>t^{\star}$, then

$$
\pi_{n}(T) \sim \sqrt{\frac{\left(R\left(t^{\star}\right)-R(0)\right) \int_{0}^{t^{\star}} \mathrm{d} s \int_{0}^{s} R(v) \mathrm{d} v}{R\left(t^{\star}\right) \int_{0}^{t^{\star}} \mathrm{d} s \int_{0}^{s} R(v) \mathrm{d} v-\frac{1}{2}\left(\int_{0}^{t^{\star}} R(v) \mathrm{d} v\right)^{2}}} \cdot \Psi\left(\frac{b+c t^{\star}}{\sqrt{2 \int_{0}^{t^{\star}} \mathrm{d} s \int_{0}^{s} R(v) \mathrm{d} v}} \sqrt{n}\right),
$$

as $n \rightarrow \infty$.

(ii) If $T=t^{\star}$, then

$$
\pi_{n}(T) \sim \frac{1}{2} \sqrt{\frac{\left(R\left(t^{\star}\right)-R(0)\right) \int_{0}^{t^{\star}} \mathrm{d} s \int_{0}^{s} R(v) \mathrm{d} v}{R\left(t^{\star}\right) \int_{0}^{t^{\star}} \mathrm{d} s \int_{0}^{s} R(v) \mathrm{d} v-\frac{1}{2}\left(\int_{0}^{t^{\star}} R(v) \mathrm{d} v\right)^{2}}} \cdot \Psi\left(\frac{b+c t^{\star}}{\sqrt{2 \int_{0}^{t^{\star}} \mathrm{d} s \int_{0}^{s} R(v) \mathrm{d} v}} \sqrt{n}\right),
$$

as $n \rightarrow \infty$.

(iii) If $T<t^{\star}$, then

$$
\pi_{n}(T) \sim \Psi\left(\frac{b+c T}{\sqrt{2 \int_{0}^{T} \mathrm{~d} s \int_{0}^{s} R(v) \mathrm{d} v}} \sqrt{n}\right),
$$

as $n \rightarrow \infty$.

Proof. Since the three cases are based on an analogous argumentation, we only treat the case $T>t^{\star}$. First we check that if $\eta(\cdot)$ is IG and satisfies IG1-IG3, then $\eta(\cdot) \in \mathbf{A 1 - A 3}$.

- In order to prove that $\mathbf{A} 1$ is satisfied it suffices to show that $\lim _{t \rightarrow \infty} \sigma_{\eta}^{2}(t) / t^{2}=0$. In view of (4.8) we have

$$
\frac{\sigma_{\eta}^{2}(t)}{t^{2}}=\frac{2 \int_{0}^{t} \mathrm{~d} s \int_{0}^{s} R(v) \mathrm{d} v}{t^{2}} \leq \frac{2 \int_{0}^{t} R(v) \mathrm{d} v}{t}=2\left(\frac{\int_{0}^{\sqrt{t}} R(v) \mathrm{d} v}{t}+\frac{\int_{\sqrt{t}}^{t} R(v) \mathrm{d} v}{t}\right) .
$$

Now A1 follows from the fact that $\int_{0}^{\sqrt{t}} R(v) \mathrm{d} v \leq R(0) \sqrt{t}$ and

$$
\lim _{t \rightarrow \infty} \frac{\int_{\sqrt{t}}^{t} R(v) d v}{t}=0
$$

the latter limit holds due to IG3.

- A2 can be proven as follows. First combine (4.8) with IG1, yielding that $\sigma_{\eta}(\cdot)$ is $C^{2}([0, \infty))$ and scrictly increasing. In order to prove concavity of $\sigma_{\eta}(\cdot)$ note that due to (4.9) it suffices to show that

$$
f(t)=2 \ddot{\sigma}_{\eta}^{2}(t) \sigma_{\eta}^{2}(t)-\left(\dot{\sigma}_{\eta}^{2}(t)\right)^{2}<0
$$


for $t>0$. This follows from $f(0)=0$ and the fact that for $t>0$

$$
f^{\prime}(t)=2 \dddot{\sigma}_{\eta}^{2}(t) \sigma_{\eta}^{2}(t)=4 R^{\prime}(t) \sigma_{\eta}^{2}(t)<0,
$$

using (4.10) and IG1-IG2.

- Following (4.8) we immediately conclude that assumption A3 is satisfied, with $\gamma \equiv 2$ and $A_{0} \equiv R(0)$ (use L'Hôpital's rule twice).

Since A1-A3 are satisfied, part (ii) of Theorem 3.2 yields

$$
\pi_{n}(T) \sim \sqrt{1+\frac{\left(\sigma_{\eta}^{\prime}\left(t^{\star}\right)\right)^{2}-\left(\sigma_{\eta}^{\prime}(0)\right)^{2}}{\sigma_{\eta}\left(t^{\star}\right) \sigma_{\eta}{ }^{\prime \prime}\left(t^{\star}\right)}} \cdot \Psi\left(\frac{b+c t^{\star}}{\sigma_{\eta}\left(t^{\star}\right)} \sqrt{n}\right),
$$

as $n \rightarrow \infty$. Using (4.9) we have

$$
\sqrt{1+\frac{\left(\sigma_{\eta}^{\prime}\left(t^{\star}\right)\right)^{2}-\left(\sigma_{\eta}{ }^{\prime}(0)\right)^{2}}{\sigma_{\eta}\left(t^{\star}\right) \sigma_{\eta}{ }^{\prime \prime}\left(t^{\star}\right)}}=\sqrt{2 \sigma_{\eta}^{2}\left(t^{\star}\right) \cdot \frac{\ddot{\sigma}_{\eta}^{2}\left(t^{\star}\right)-2\left(\sigma_{\eta}^{\prime}(0)\right)^{2}}{2 \ddot{\sigma}_{\eta}^{2}\left(t^{\star}\right) \sigma_{\eta}^{2}\left(t^{\star}\right)-\left(\dot{\sigma}_{\eta}^{2}\right)^{2}}} .
$$

Now from (4.10) and the fact that $\left(\sigma_{\eta}{ }^{\prime}(0)\right)^{2}=R(0)$, we obtain

$$
\pi_{n}(T) \sim \sqrt{\frac{\left(R\left(t^{\star}\right)-R(0)\right) \int_{0}^{t^{\star}} \mathrm{d} s \int_{0}^{s} R(v) \mathrm{d} v}{R\left(t^{\star}\right) \int_{0}^{t^{\star}} \mathrm{d} s \int_{0}^{s} R(v) \mathrm{d} v-\frac{1}{2}\left(\int_{0}^{t^{\star}} R(v) \mathrm{d} v\right)^{2}}} \cdot \Psi\left(\frac{b+c t^{\star}}{\sqrt{2 \int_{0}^{t^{\star}} \mathrm{d} s \int_{0}^{s} R(v) \mathrm{d} v}} \sqrt{n}\right),
$$

as $n \rightarrow \infty$. This completes the proof.

Combining Theorem 3.5 with Proposition 4.3 we obtain the exact asymptotics of the stationary buffer overflow probability.

Proposition 4.4 Let $\eta(\cdot)$ be IG input such that IG1-IG3 are satisfied. Then

$$
\pi_{n} \sim \sqrt{\frac{\left(R\left(t^{\star}\right)-R(0)\right) \int_{0}^{t^{\star}} \mathrm{d} s \int_{0}^{s} R(v) \mathrm{d} v}{R\left(t^{\star}\right) \int_{0}^{t^{\star}} \mathrm{d} s \int_{0}^{s} R(v) \mathrm{d} v-\frac{1}{2}\left(\int_{0}^{t^{\star}} R(v) \mathrm{d} v\right)^{2}}} \cdot \Psi\left(\frac{b+c t^{\star}}{\sqrt{2 \int_{0}^{t^{\star}} \mathrm{d} s \int_{0}^{s} R(v) \mathrm{d} v}} \sqrt{n}\right),
$$

as $n \rightarrow \infty$.

\section{Proofs}

In this section we present the proofs of Theorems 3.2-3.5. In the following by the attached bar we always mean the standardized process, that is $\bar{X}(t)=X(t) / \sigma_{X}(t)$ for some Gaussian process $X(\cdot)$. Moreover let

$$
\mathcal{H}_{\alpha}^{R}:=\lim _{S \rightarrow \infty} \mathbb{E} \exp \left(\sup _{t \in[-S, S]} B_{\alpha / 2}(t)-(1+R) t^{\alpha}\right),
$$

and

$$
\mathcal{F}_{\alpha}^{R}:=\lim _{S \rightarrow \infty} \mathbb{E} \exp \left(\sup _{t \in[0, S]} B_{\alpha / 2}(t)-(1+R) t^{\alpha}\right) .
$$

The idea of the proofs of Theorems 3.2, 3.3 and 3.4 is based on an appropriate use of Theorem 1 in Piterbarg \& Prisyazhnyuk [23]. Since this result plays the crucial role in the following analysis, we present it in the form that is suitable for us. 
Theorem 5.1 Let $(\xi(t))_{t \in[0, T]}$ be a centered Gaussian process with continuous sample paths a.s. and variance function $\sigma_{\xi}^{2}(\cdot)$ such that the maximum of $\sigma_{\xi}(\cdot)$ on $[0, T]$ is attained at a unique point $t^{\star}$ with $\sigma_{\xi}\left(t^{\star}\right)=1$. Make the following assumptions:

(a) $\sigma_{\xi}(\cdot)$ is polynomial in a neighborhood of $t^{\star}$ : there exist $A, \beta>0$ such that

$$
1-\sigma_{\xi}\left(t+t^{\star}\right)=A|t|^{\beta}(1+o(1)) \quad \text { as } t \rightarrow 0 ;
$$

(b) Local stationarity: there exist $D, \alpha>0$ such that

$$
1-\operatorname{Cov}(\bar{\xi}(t), \bar{\xi}(s))=D|t-s|^{\alpha}+o\left(|t-s|^{\alpha}\right) \quad \text { as } s, t \rightarrow t^{\star}
$$

(c) Regularity: there exist $C, \alpha_{1}>0$ such that, for $s, t \in[0, T]$,

$$
\mathbb{E}(\xi(t)-\xi(s))^{2} \leq C|t-s|^{\alpha_{1}} .
$$

Then,

(i) if $\beta>\alpha$, with $\mathcal{G}_{\alpha, \beta}:=\mathcal{H}_{\alpha} \Gamma(1 / \beta) D^{1 / \alpha} \beta^{-1} A^{-1 / \beta}$, as $u \rightarrow \infty$,

$$
\mathbb{P}\left(\sup _{t \in[0, T]} \xi(t)>u\right) \sim u^{\frac{2}{\alpha}-\frac{2}{\beta}} \Psi(u) \cdot \begin{cases}2 \cdot \mathcal{G}_{\alpha, \beta} & \text { if } t^{\star} \in(0, T) ; \\ \mathcal{G}_{\alpha, \beta} & \text { if } t^{\star}=0 \text { or } t^{\star}=T ;\end{cases}
$$

(ii) if $\beta=\alpha$, with $R:=A / D$, as $u \rightarrow \infty$,

$$
\mathbb{P}\left(\sup _{t \in[0, T]} \xi(t)>u\right) \sim \Psi(u) \cdot \begin{cases}\mathcal{H}_{\alpha}^{R} & \text { if } t^{\star} \in(0, T) \\ \mathcal{F}_{\alpha}^{R} & \text { if } t^{\star}=0 \text { or } t^{\star}=T\end{cases}
$$

(iii) if $\beta<\alpha$, as $u \rightarrow \infty$,

$$
\mathbb{P}\left(\sup _{t \in[0, T]} \xi(t)>u\right) \sim \Psi(u)
$$

Before presinting the proofs of Theorems 3.2-3.5 we give some technical lemmas.

Lemma 5.2 Let $\eta(\cdot) \in \mathbf{A 2}$. Then, for each $T \in\left[0, t^{\star}\right)$,

(i) $1-\frac{m\left(t^{\star}\right)}{m\left(t+t^{\star}\right)}=-\frac{\sigma_{\eta}{ }^{\prime \prime}\left(t^{\star}\right)}{2 \sigma_{\eta}\left(t^{\star}\right)} \cdot t^{2} \cdot(1+o(1))$ as $t \rightarrow 0$;

(ii) $1-\frac{m(T)}{m(T-t)}=-\frac{m^{\prime}(T)}{m(T)} \cdot t \cdot(1+o(1))$ as $t \uparrow T$.

Proof. First we prove (i). Because of A2, we can represent $\sigma_{\eta}(\cdot)$ around $t^{\star}$ by a second order Taylor expansion:

$$
\begin{aligned}
1-\frac{m\left(t^{\star}\right)}{m\left(t+t^{\star}\right)} & =\frac{\sigma_{\eta}\left(t^{\star}\right)\left(b+c\left(t+t^{\star}\right)\right)-\sigma_{\eta}\left(t+t^{\star}\right)\left(b+c t^{\star}\right)}{\sigma_{\eta}\left(t^{\star}\right)\left(b+c\left(t+t^{\star}\right)\right)} \\
& =\frac{\sigma_{\eta}\left(t^{\star}\right)\left(b+c\left(t+t^{\star}\right)\right)-\left(\sigma_{\eta}\left(t^{\star}\right)+\sigma_{\eta}{ }^{\prime}\left(t^{\star}\right) t+\sigma_{\eta}{ }^{\prime \prime}\left(t^{\star}+\theta(t)\right) \frac{t^{2}}{2}\right)\left(b+c t^{\star}\right)}{\sigma_{\eta}\left(t^{\star}\right)\left(b+c\left(t+t^{\star}\right)\right)} \\
& =-\frac{\sigma_{\eta}{ }^{\prime \prime}\left(t^{\star}+\theta(t)\right)}{2 \sigma_{\eta}\left(t^{\star}\right)} \cdot \frac{b+c t^{\star}}{b+c\left(t+t^{\star}\right)} \cdot t^{2}
\end{aligned}
$$


where $\theta(t) \in[0, t]$, and (5.11) is due to Remark 3.1. Hence, taking $t \rightarrow 0$, we complete the proof of (i).

Similarly, to prove (ii) notice that

$$
\begin{aligned}
1-\frac{m(T)}{m(T-t)} & =\frac{\sigma_{\eta}(T) \cdot(b+c(T-t))-\sigma_{\eta}(T-t) \cdot(b+c T)}{\sigma_{\eta}(T)(b+c(T-t))} \\
& =\frac{\sigma_{\eta}(T) \cdot(b+c(T-t))-\left(\sigma_{\eta}(T)-\sigma_{\eta}{ }^{\prime}(T+\theta(t)) t\right) \cdot(b+c T)}{\sigma_{\eta}(T)(b+c(T-t))} \\
& =-\frac{\sigma_{\eta}(T) c-(b+c T) \cdot \sigma_{\eta}^{\prime}(T+\theta(t))}{\sigma_{\eta}(T)(b+c(T-t))} \cdot t
\end{aligned}
$$

where $\theta(t) \in[0, t]$. Now notice that

$$
\frac{\sigma_{\eta}(T) c-(b+c T) \cdot \sigma_{\eta}{ }^{\prime}(T)}{\sigma_{\eta}(T)(b+c T)}=\frac{m^{\prime}(T)}{m(T)}
$$

and $m^{\prime}(T)<0$ for $T<t^{\star}$ (apply Lemma 3.1). This completes the proof.

Lemma 5.3 Let $\eta(\cdot) \in \mathbf{A 2 - A 3}$. Then

(i) If $\gamma<2$, then, for each $T>0$,

$$
1-\operatorname{Cov}(\bar{\eta}(s), \bar{\eta}(t))=\frac{A_{0}}{2 \sigma_{\eta}^{2}(T)}|t-s|^{\gamma}+o\left(|t-s|^{\gamma}\right) \quad \text { as } s, t \rightarrow T ;
$$

(ii) if $\gamma=2$, then, for each $T>0$,

$$
1-\operatorname{Cov}(\bar{\eta}(s), \bar{\eta}(t))=\frac{\left(\sigma_{\eta}{ }^{\prime}(0)\right)^{2}-\left(\sigma_{\eta}{ }^{\prime}(T)\right)^{2}}{2 \sigma_{\eta}^{2}(T)}|t-s|^{2}+o\left(|t-s|^{2}\right) \quad \text { as } s, t \rightarrow T .
$$

Proof. The case (i) is due to Lemma 3.2 in Dębicki \& Rolski [10]. It follows directly from A3 and

$$
\begin{aligned}
& \lim _{s, t \rightarrow T} \frac{1-\operatorname{Cov}(\bar{\eta}(s), \bar{\eta}(t))}{|t-s|^{\gamma}}= \\
& \quad=\lim _{s, t \rightarrow T}\left(\frac{\sigma_{\eta}^{2}(t-s)}{2 \sigma_{\eta}(s) \sigma_{\eta}(t)}-\frac{\left(\sigma_{\eta}(t)-\sigma_{\eta}(s)\right)^{2}}{2 \sigma_{\eta}(t) \sigma_{\eta}(s)}\right)|t-s|^{-\gamma} \\
& \quad=\lim _{s, t \rightarrow T}\left(\frac{\sigma_{\eta}^{2}(t-s)}{2 \sigma_{\eta}(s) \sigma_{\eta}(t)}\right)|t-s|^{-\gamma}=\frac{A_{0}}{2 \sigma_{\eta}^{2}(T)},
\end{aligned}
$$

assuming, without loss of generality, $t>s$.

Notice that this reasoning does not go through for $\gamma=2$, as in that case

$$
\lim _{s, t \rightarrow T} \frac{\left(\sigma_{\eta}(t)-\sigma_{\eta}(s)\right)^{2}}{2 \sigma_{\eta}(t) \sigma_{\eta}(s)}|t-s|^{-2}=\frac{\left(\sigma_{\eta}{ }^{\prime}(T)\right)^{2}}{2 \sigma_{\eta}^{2}(T)}>0 .
$$

In order to prove the case (ii) note that

$$
1-\operatorname{Cov}(\bar{\eta}(s), \bar{\eta}(t))=\frac{\sigma_{\eta}^{2}(|t-s|)-\left(\sigma_{\eta}(t)-\sigma_{\eta}(s)\right)^{2}}{2 \sigma_{\eta}(t) \sigma_{\eta}(s)} .
$$


Thus

$$
\begin{aligned}
\lim _{s, t \rightarrow T} \frac{1-\operatorname{Cov}(\bar{\eta}(s), \bar{\eta}(t))}{|t-s|^{2}} & =\frac{1}{2 \sigma_{\eta}^{2}(T)} \lim _{s, t \rightarrow T} \frac{\sigma_{\eta}^{2}(|t-s|)-\left(\sigma_{\eta}(t)-\sigma_{\eta}(s)\right)^{2}}{|t-s|^{2}} \\
& =\frac{\left(\sigma_{\eta}{ }^{\prime}(0)\right)^{2}-\left(\sigma_{\eta}{ }^{\prime}(T)\right)^{2}}{2 \sigma_{\eta}^{2}(T)} .
\end{aligned}
$$

This completes the proof.

Lemma 5.4 Let $\eta(\cdot) \in \mathbf{A 2}$. Then for each $T>0$ there exist constants $G_{T}>0$ and $\alpha_{1}>0$ such that

$$
\mathbb{E}\left(\bar{\eta}(t) \frac{m\left(t^{\star}\right)}{m(t)}-\bar{\eta}(s) \frac{m\left(t^{\star}\right)}{m(s)}\right)^{2} \leq G_{T} \cdot|t-s|^{\alpha_{1}}
$$

for all $s, t \in[0, T]$.

Proof. Let $T>0$ be given. It is straightforward to show that the left hand side of (5.13) equals

$$
m^{2}\left(t^{\star}\right) \mathbb{E}\left(\frac{\eta(t)}{c t+b}-\frac{\eta(s)}{c s+b}\right)^{2}=\frac{m^{2}\left(t^{\star}\right)}{(b+c t)^{2}(b+c s)^{2}} \mathbb{E}((c s+b) \eta(t)-(b+c t) \eta(s))^{2} .
$$

This expression is majorized as follows:

$$
\begin{aligned}
& \leq \frac{m^{2}\left(t^{\star}\right)}{b^{4}} \mathbb{E}((\eta(t)-\eta(s))(b+c s)-\eta(s)(c t-c s))^{2} \\
& \leq \frac{m^{2}\left(t^{\star}\right)}{b^{4}}\left(2 \mathbb{E}((\eta(t)-\eta(s))(b+c s))^{2}+2 \mathbb{E}(\eta(s)(c t-c s))^{2}\right) \\
& =\frac{m^{2}\left(t^{\star}\right)}{b^{4}}\left(2 \sigma_{\eta}^{2}(|t-s|)(b+c s)^{2}+2 \sigma_{\eta}^{2}(s) c^{2}|t-s|^{2}\right),
\end{aligned}
$$

where (5.14) follows from the fact that $(x-y)^{2} \leq 2 x^{2}+2 y^{2}$ for all $x, y$. Thus from A2, for $\alpha_{1}=\min (\gamma, 2)$ we have that there exists a constant $G_{T}>0$ such that the above is not larger than $G_{T} \cdot|t-s|^{\alpha_{1}}$ for all $s, t \in[0, T]$.

We will now present the basic idea behind our proof. By a number of simple transformations we translate the probabilities $\pi_{n}$ and $\pi_{n}(T)$ into the framework of Theorem 5.1. We will focus on the transient probabilities $\pi_{n}(T)$; the stationary probabilities $\pi_{n}$ are treated similarly. Observe that

$$
\begin{aligned}
\pi_{n}(T) & =\mathbb{P}\left(\sup _{t \in[0, T]} \sum_{i=1}^{n} \eta_{i}(t)-n c t>n b\right) \\
& =\mathbb{P}\left(\sup _{t \in[0, T]} \frac{\sum_{i=1}^{n} \eta_{i}(t)}{\sqrt{n}} \cdot \frac{1}{b+c t}>\sqrt{n}\right) \\
& =\mathbb{P}\left(\sup _{t \in[0, T]} \eta(t) \cdot \frac{1}{b+c t}>\sqrt{n}\right) \\
& =\mathbb{P}\left(\sup _{t \in[0, T]} \frac{\eta(t)}{\sigma_{\eta}(t)} \cdot \frac{m\left(t^{\star}\right)}{m(t)}>m\left(t^{\star}\right) \sqrt{n}\right),
\end{aligned}
$$


were $(5.15)$ follows from the fact that $\eta_{i}(\cdot)$ are Gaussian i.i.d. copies of $\eta(\cdot)$. Let

$$
\mu(t):=\frac{\eta(t)}{\sigma_{\eta}(t)} \cdot \frac{m\left(t^{\star}\right)}{m(t)} .
$$

Notice that we are in the framework of Theorem 5.1: the process $\mu(\cdot)$ has standard deviation function $\sigma_{\mu}(\cdot)$ with $\sigma_{\mu}(t)=m\left(t^{\star}\right) / m(t)$; evidently, $\sigma_{\mu}^{2}(\cdot)$ has $t^{\star}$ as unique maximizer (see Lemma 3.1) and $\max _{t \in[0, T]} \sigma_{\mu}(t)=1$. So, to prove Theorems 3.2-3.4, we have to check if Assumptions (a), (b), and (c) of Theorem 5.1 apply.

\subsection{Proof of Theorem 3.2}

Noting that

$$
\operatorname{Cov}(\bar{\mu}(s), \bar{\mu}(t))=\operatorname{Cov}(\bar{\eta}(s), \bar{\eta}(t)),
$$

and following Lemmas 3.1, 5.2, and 5.3, we infer that Assumptions (a) and (b) of Theorem 5.1 are satisfied with $\alpha \equiv \gamma, \beta \equiv 2$, and $A \equiv-\sigma_{\eta}{ }^{\prime \prime}\left(t^{\star}\right) / 2 \sigma_{\eta}\left(t^{\star}\right)$. Moreover from Lemma 5.4 it follows that Assumption (c) is satisfied.

- If $0<\gamma<2$, then Lemma 5.3 states that $D \equiv A_{0} / 2 \sigma_{\eta}^{2}\left(t^{\star}\right)$. Since $T>t^{\star}$, Theorem 5.1 gives the result immediately, using that $\Gamma(1 / 2)=\sqrt{\pi}$.

- If $\gamma=2$, then from Lemma 5.3

$$
D \equiv \frac{\left(\sigma_{\eta}{ }^{\prime}(0)\right)^{2}-\left(\sigma_{\eta}{ }^{\prime}(T)\right)^{2}}{2 \sigma_{\eta}^{2}(T)} .
$$

Thus, using an argumentation similar to the case $0<\gamma<2$, we obtain

$$
\mathbb{P}\left(\sup _{t \in[0, T]} \mu(t)>m\left(t^{\star}\right) \sqrt{n}\right) \sim \Psi\left(m\left(t^{\star}\right) \sqrt{n}\right) \cdot \mathcal{H}_{\gamma}^{R},
$$

where

$$
R \equiv-\left(\frac{\sigma_{\eta}^{\prime \prime}\left(t^{\star}\right)}{2 \sigma_{\eta}\left(t^{\star}\right)}\right) \cdot\left(\frac{\left(\sigma_{\eta}{ }^{\prime}(0)\right)^{2}-\left(\sigma_{\eta}{ }^{\prime}(T)\right)^{2}}{2 \sigma_{\eta}^{2}(T)}\right)^{-1}=\frac{\sigma_{\eta}\left(t^{\star}\right) \sigma_{\eta}{ }^{\prime \prime}\left(t^{\star}\right)}{\left.\left(\sigma_{\eta^{\prime}}{ }^{\star} t^{\star}\right)\right)^{2}-\left(\sigma_{\eta}{ }^{\prime}(0)\right)^{2}}
$$

Now notice that $\mathcal{H}_{\gamma}^{R}=\sqrt{1+R^{-1}}$, according to Konstant \& Piterbarg [13]. This concludes our proof.

\subsection{Proof of Theorem 3.3}

The proof of Theorem 3.3 is completely analogous to the proof of Theorem 3.2, except that $t^{\star}=T$. 


\subsection{Proof of Theorem 3.4}

The idea of the proof of Theorem 3.4 is analogous to the proof of Theorem 3.2 and thus we present only the main steps of argumentation.

We define the process $\mu_{T}(\cdot)$ as follows:

$$
\mu_{T}(t):=\frac{\eta(t)}{\sigma_{\eta}(t)} \cdot \frac{m(T)}{m(t)} .
$$

By $\sigma_{\mu_{T}}(\cdot)$ we denote the standard deviation function of $\mu_{T}(\cdot)$. Following the same argumentation as presented in the proof of Theorem 3.2, we write

$$
\pi_{n}(T)=\mathbb{P}\left(\sup _{t \in[0, T]} \mu(t)>m(T) \sqrt{n}\right) .
$$

Since $T<t^{\star}$, Lemma 3.1 states that $\sigma_{\mu_{T}}(\cdot)$ attains its maximum in the interval $[0, T]$ at $t=T$; with $\sigma_{\mu_{T}}(T)=1$. Due to Lemmas 5.2 and 5.3 process $\mu_{T}(\cdot)$ satisfies conditions (a) and (b) of Theorem 5.1 with $\alpha \equiv \gamma, \beta \equiv 1, A \equiv-m^{\prime}(T) / m(T)$. Moreover, from Lemma 5.4 it follows that condition $(c)$ is fulfilled.

- If $\gamma<1$, then Lemma 5.3 gives $D \equiv A_{0} / 2 \sigma_{\eta}^{2}(T)$. Using (i) of Theorem 5.1 we get part (i) of Theorem 3.4.

- If $\gamma=1$, again from Lemma $5.3, D \equiv A_{0} / 2 \sigma_{\eta}^{2}(T)$. Using (ii) of Theorem 5.1 we get

$$
\mathbb{P}\left(\sup _{t \in[0, T]} \mu(t)>m(T) \sqrt{n}\right) \sim \Psi(m(T) \sqrt{n}) \cdot \mathcal{F}_{1}^{R},
$$

where $R:=-\left(m^{\prime}(T) / m(T)\right) \cdot\left(2 \sigma_{\eta}^{2}(T) / A_{0}\right)$. Moreover,

$$
\begin{aligned}
\mathcal{F}_{1}^{R} & =1+\int_{0}^{\infty} \mathbb{P}\left(\sup _{t \in[0, \infty)} \sqrt{2} B_{1 / 2}(t)-(1+R) t>u\right) e^{u} \mathrm{~d} u \\
& =1+\int_{0}^{\infty} \mathbb{P}\left(\sup _{t \in[0, \infty)} B_{1 / 2}(t)-\frac{(1+R)}{\sqrt{2}} t>\frac{u}{\sqrt{2}}\right) e^{u} \mathrm{~d} u \\
& =1+\int_{0}^{\infty} \exp (-u(1+R)) e^{u} \mathrm{~d} u=1+\frac{1}{R},
\end{aligned}
$$

where (5.16) follows from

$$
\mathbb{P}\left(\sup _{t \in[0, \infty)} B_{1 / 2}(t)-c t>u\right)=e^{-2 c u}
$$

for each $c, u>0$. This completes the proof of (ii) of Theorem 3.4.

- If $\gamma>1$, then the stated follows directly from (iii) of Theorem 5.1. 


\subsection{Proof of Theorem 3.5}

Since for each $T>0, n \geq 0$ we have $\pi_{n}(T) \leq \pi_{n}$, then it is enough to show that

$$
\limsup _{n \rightarrow \infty} \pi_{n} / \pi_{n}(T) \leq 1
$$

for some $T>t^{\star}$.

Note that, using the same argumentation as in the proof of Theorem 3.2,

$$
\pi_{n}=\mathbb{P}\left(\sup _{t \in[0, \infty)} \frac{\eta(t)}{\sigma_{\eta}(t)} \cdot \frac{m\left(t^{\star}\right)}{m(t)}>m\left(t^{\star}\right) \sqrt{n}\right) .
$$

Using the union bound we have

$$
\pi_{n} \leq \pi_{n}(T)+\mathbb{P}\left(\sup _{t \in[T, \infty)} \frac{\eta(t)}{\sigma_{\eta}(t)} \cdot \frac{m\left(t^{\star}\right)}{m(t)}>m\left(t^{\star}\right) \sqrt{n}\right) .
$$

Due to Lemma 3.1 there exists a $T>t^{\star}$ such that $m(t)<m\left(t^{\star}\right) / 2$ for $t>T$, yielding

$$
\begin{aligned}
\mathbb{P}\left(\sup _{t \in[T, \infty)} \frac{\eta(t)}{\sigma_{\eta}(t)} \cdot \frac{m\left(t^{\star}\right)}{m(t)}>m\left(t^{\star}\right) \sqrt{n}\right) & \leq \mathbb{P}\left(\sup _{t \in[T, \infty)} \frac{\eta(t)}{\sigma_{\eta}(t)}>2 m\left(t^{\star}\right) \sqrt{n}\right) \\
& \leq 2 \Phi\left(2 m\left(t^{\star}\right) \sqrt{n}-K\right)=o\left(\pi_{n}(T)\right)
\end{aligned}
$$

as $n \rightarrow \infty$, where (5.17) follows from the Borell inequality (see e.g. Theorem D.1 in [22]), and $K$ is a constant. This completes the proof.

\section{References}

[1] Addie, R., Mannersalo, P., \& Norros, I. (1999) Performance formulae for queues with Gaussian input. Proceedings of ITC 16, 1169-1178.

[2] Anick, D., Mitra, D., \& Sondhi, M.M. (1982) Stochastic theory of a data handling system with multiple sources. Bell System Technical Journal 61, 1871-1894.

[3] Bahadur, R.R. \& Rao, R.R. (1960) On deviations of the sample mean. The Annals of Mathematical Statistics 31, 1015-1027.

[4] Botvich, D. \& Duffield, N. (1995) Large deviations, the shape of the loss curve, and economies of scale in large multiplexers. Queueing Systems 20, 293-320.

[5] Courcoubetis, C. \& Weber, R. (1996) Buffer overflow asymptotics for a buffer handling many traffic sources. Journal of Applied Probability 33, 886-903.

[6] Dębicki, K (2002) Ruin probabilities for Gaussian integrated processes. Stoch. Proc. Appl. 98, 151-174.

[7] Dębicki, K. \& Palmowski, Z. (1999) Heavy traffic asymptotics of on-off fluid model. Queuing Systems 33, 327-338.

[8] Dẹbicki, K. \& Rolski, T. (1995) A Gaussian fluid model. Queueing Systems 20, 433-452. 
[9] Dębicki, K. \& Rolski, T. (2000) Gaussian fluid models; a survey. Symposium on Performance Models for Information Communication Networks. Sendai, Japan, January 23-25,2000. Available at

http://www.math.uni.wroc.pl/ rolski/publications.html.

[10] Dębicki, K. \& Rolski, T. (2001) A note on transient Gaussian fluid models. Accepted for publication in Queueing Systems. Available at

http://www.math.uni.wroc.pl/ rolski/publications.html.

[11] Duffield, N. \& O'Connell, N. (1995) Large deviations and overflow probabilities for the general single server queue, with applications. Proceedings of the Cambridge Philosophical Society 118, 363-374.

[12] Hüsler, J. \& Piterbarg, V.I. (1999) Extremes of a certain class of Gaussian processes. Stochastic Processes and their Applications 83, 257-271.

[13] Konstant, D.G. \& Piterbarg, V. (1993) Extreme values of the cyclostationary Gaussian random processes. Journal of Applied Probability 30, 82-97.

[14] Kulkarni, V. \& Rolski, T. (1994). Fluid model driven by an Ornstein-Uhlenbeck process. Probability in the Engineering and Informational Sciences 8, 403-417.

[15] Leland, W.E., Taqqu, M.S., Willinger, W., \& Wilson, D.V. (1994) On the self-similar nature of Ethernet traffic. IEEE/ACM Transactions on Networking 2, 1-15.

[16] Likhanov, N. \& Mazumdar, R. (1999) Cell loss asymptotics in buffers fed with a large number of independent stationary sources. Journal of Applied Probability 36, 86-96.

[17] Mandjes, M. \& Borst, S. (2000) Overflow behavior in queues with many long-tailed inputs. Advances in Applied Probability 32, 1150-1167.

[18] Mandjes, M. \& Kim, J.H. (2001). Large deviations for small buffers: an insensitivity result. Queueing Systems 37, 349-362.

[19] Massoulié, L. \& Simonian, A. (1999) Large buffer asymptotics for the queue with FBM input. Journal of Applied Probability 36, 894-906.

[20] Narayan, O. Exact asymptotic queue length distribution for fractional Brownian traffic. $A d-$ vances in Performance Analysis 1, 39-63.

[21] Norros, I. (1994) A storage model with self-similar input. Queuing Systems 16, 387-396.

[22] Piterbarg, V.I. (1996) Asymptotic methods in the theory of Gaussian processes and fields. Translations of Mathematical Monographs 148, AMS, Providence.

[23] Piterbarg, V.I. \& Prisyazhnyuk, V. (1978) Asymptotic behavior of the probability of a large excursion for a nonstationary Gaussian processes. Theory of Probability and Mathematical Statistics. 18, 121-133.

[24] Shorack, G.R. \& Wellner, J.A. (1986) Empirical processes with applications to statistics. Wiley, New York. 
[25] Simonian, A. \& Guibert, J. (1995) Large deviations approximation for fluid queues fed by a large number of on/off sources. IEEE Journal of Selected Areas in Communications 13, $1017-1027$.

[26] Simonian, A. \& Virtamo, J. (1991) Transient and stationary distributions for fluid queue and input processes with density. SIAM Journal of Applied Mathematics 51, 1731-1739.

[27] Weiss, A. (1986). A new technique of analyzing large traffic systems. Advances in Applied Probability 18, 506-532. 